Cell Research (2001); 11(2):161-163

SHORT COMMUNICATION

\title{
GABA transporter 1 transcriptional starting site exhibiting tissue specific difference
}

\author{
JIN XiAo Ping*, FAng HUANG*, Na YANG, Bu Feng LU, Jian FEI **, Li He GUO** \\ Institute of Biochemistry and Cell Biology, Shanghai Institutes for Biological Science, Chinese Academy of Sciences, \\ 320 Yueyang Road, Shanghai 200031, China
}

\begin{abstract}
GABA transporter 1(GAT1) takes important roles in multiple physiological processes through the uptake and release of GABA, but the regulation of GAT1 gene expression in different tissues is rarely known. To address the question, first, 5' Rapid amplification of cDNA end (RACE) was used to determine GAT1 transcriptional starting sites in neonatal mouse cerebral cortex and intestine, adult mouse brain and adult rat testis. The products of 5' RACE were confirmed by DNA sequencing. We found that the transcript of GAT1 in neonatal mouse cerebral cortex and adult mouse brain starts at the same site (inside of exon 1), while in mouse intestine, GAT1 starts transcription in intron 1, and in rat testis, the transcript of GAT1 has an additional untranslation exon to the 5' direction.
\end{abstract}

Key words: GABA transporter 1(GAT1), transcriptional starting site, 5' RACE, tissue specific difference, DNA sequencing.

\section{INTRODUCTION}

In the vertebrate central nervous system (CNS), GABA transporters (GAT) are believed to play an important role in termination of GABAergic transmission. GAT1 was the first cloned member of neurotransmitter transporters superfamily[1], and soon, other three subtypes (GAT2-4) were subsequently cloned. Since GABA is the predominant inhibitory neurotransmitter in CNS, abnormality of GATs has a direct relationship with certain kinds of nervous disorders, such as epilepsy and cognitive deterioration. Recent studies indicated that GAT1 also expresses in rat testis and is involved in the acrosome reaction[2], [3]. GAT1 expresses widely in nervous system and non-nervous tissues, and takes part in different physiological processes. In order to study the regulation of GAT1 gene expression in normal tissues and/or under pathologi-

\footnotetext{
* The two authors contributed equally to the research. ** Corresponding authors, Email: mhzhang@sunm.shcnc.ac.cn Received Feb-15-2001 Revised April-26-2001 Accepted April-272001
}

cal conditions, first, we determined the transcriptional starting sites of GAT1 by the method of $5^{\prime}$ RACE (rapid amplification of cDNA ends, an efficient method to find out the starting of a transcript) in neonatal mouse cerebral cortex and intestine, adult mouse brain and adult rat testis.

\section{MATERIALS AND METHODS}

\section{Primers}

Primer P1 (5' CCGCAAAGATGAGCGTCAGGAAATATGG 3’ , 421-394 in mouse GAT1 cDNA, GenBank No. M92378), primer P2 (5'” AGCACAAACTTGGCAGCG 3' , 134-117), P1 and $\mathrm{P} 2$ are conserved in mouse and rat GAT1 cDNA with only one nucleotide difference in P2. Primer P3 (5, GAGTCTTCTCCTAAAGGCTTTCTCC 3', 54-20 in rat GAT1 cDNA, GenBank No. M59742) and primer P4 (5, GAGGACACTGTGGTCACGAAAGAGC 3', , 2-27 in rat testis GAT1 cDNA, GenBank No. AF332363).

\section{Genomic DNA and mRNA isolation}

\footnotetext{
Rat genomic DNA was isolated from adult rat liver according to the standard procedure [4].Total RNA was isolated from Day 1 mouse cerebral cortex (Balb/C, within 24-36 h postnatal), Day 3 mouse intestine (within 72-96 hours postnatal), adult mouse brain (one year old), and adult rat testis (Wistar, four months old)
} 
using Trizol reagent (GIBCO). RNase-free DNase I (Promega) was used to eliminate the contamination of chromosomal DNA. mRNA was purified from RNase-free DNase I pretreated total RNA using OligotexTM mRNA Mini Kit (QIAGENE).

\section{5' RACE (rapid amplification of cDNA ends)}

The first strand cDNA synthesis was processed as the recommendation of RACE Kit supplier (Clontech). For 5' RACE, primer P1 was used as GAT1 specific primer in the first round of PCR amplification, and primer P2 was used as nested specific primer in the second round of amplification. 5, RACE products were cloned to T-vector (Promega) and automatically sequenced on ABI 310. The sequence data were analyzed with GCG software (http://www.biosino.org) and deposited in GenBank.

\section{RESULTS AND DISCUSSION}

\section{GAT1 transcriptional starting sites in neonatal} mouse cerebral cortex and adult mouse brain

Expression of GAT1 was detected in rat cerebrum and cerebellum [1]. By means of 5' RACE using primer P1, P2 (indicated in Fig 2) and sequence analysis, we found that the GAT1 transcriptional starting site in neonatal mouse cerebral cortex (GenBank No. AF332360) and adult mouse brain (GenBank No. AF332362) was exactly the same, inside of exon I, 12 base pairs downstream of published mouse GAT1 cDNA sequence (GenBank No. M92378) (Fig 1a and Fig 2).

\section{GAT1 transcriptional starting site in neonatal} mouse intestine

The same 5' RACE strategy was used to deter- mine the transcriptional starting site in neonatal mouse intestine. Different from mouse brain, transcription of GAT1 gene in Day3 intestine starts in intron 1[5],[6], $628 \mathrm{bp}$ upstream of exon II (GenBank No. AF332361)(Fig 1a and Fig 2).

\section{GAT1 transcriptional starting site in rat testis}

Recent findings demonstrated that GAT1 also expresses in rat testis, and is involved in the acrosome reaction, an essential fertilization event, and that overexpression of GAT1 in transgenic mice affects the testicular morphology[2],[3]. The same primers (P1 and P2) were used to identify GAT1 transcriptional starting site in adult rat testis. By cloning and sequencing the 5' RACE product, we found that there is an additional untranslation sequence extending to the 5' direction of rat GAT1 cDNA (306 bp in length, GenBank No. AF332363). To find the corresponding part of this 306bp fragment in the rat genome, primer P3 and P4 (indicated in Fig 3) were used to amplify rat genomic DNA by PCR, and a band, 1121 bp in length, was amplified (GenBank No. AF332364). Comparing the sequence data, we knew that this 306bp fragment is encoded by one exon (named as exon 1 ), and there exists an intron between exon 1 and exon $2,783 \mathrm{bp}$ in size with typical starting nucleotides-gt... and ending nucleotides-....ag. Exon 2 starts at the 13th nucleotide of published rat GAT1 cDNA sequence (GenBank No. M59742), and the 12 nucleotides at 5 ' terminal could not be found in a

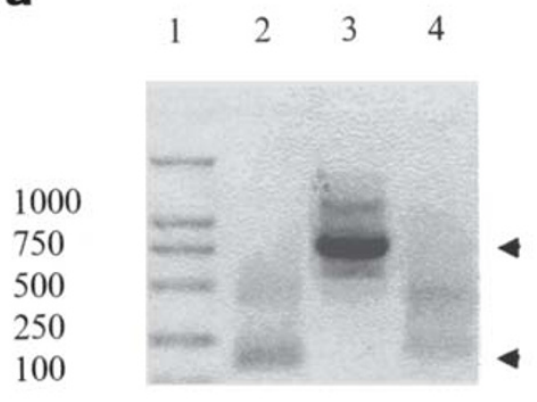

b

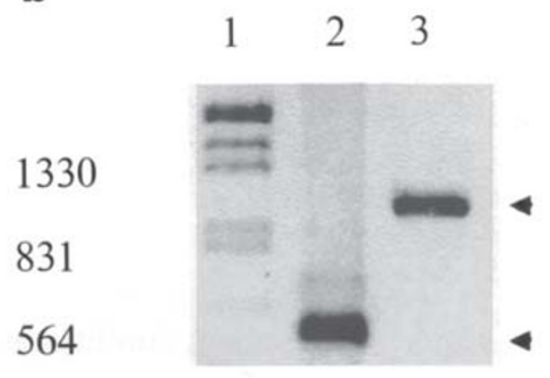

Fig 1. PCR products of 5' RACE for GAT1 in different tissues 1a, 5' RACE products in mouse. Lane 1: DNA Marker; Lane 2: Day 1 cerebral cortex; Lane 3: Day 3 intestine; Lane 4: Adult brain. 1b, 5' RACE product in rat testis and corresponding fragment in the rat genome. Lane 1: DNA Marker; Lane 2: 5' RACE product in rat testis; Lane 3: PCR amplified fragment in rat genomic DNA using primers P3 and P4. Arrows indict bands corresponding to GAT1. 
the sequence of $1121 \mathrm{bp}$ fragment we obtained from rat genomic DNA (Fig 1 b and Fig 3 ).

GABA is a multifunctional molecule in vertebrates. GABA transporters can regulate the biological functions of GABA by controlling the intensity and duration of its action. GAT1 mainly expressed in brain, and to less extent, in spleen and testis, but its expression cannot be detected in kidney, liver and heart. We also tested 5' RACE of

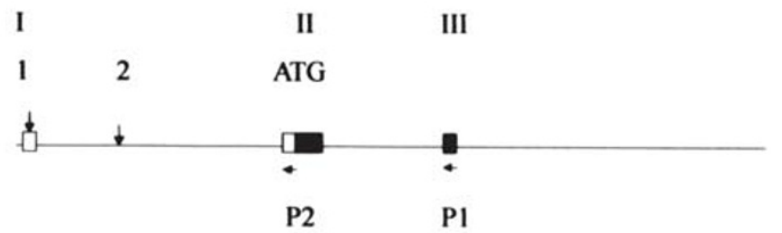

Starting site 1: 5' GGAGAAAGCCTTTAGG......3,

Starting site 2: 5' AAGTACATCAACATGA......3'

Fig 2. Intron and exon distribution of 5' region of mouse GAT1 gene Boxes in white and black represent exons (I, II and III). Vertical arrow 1 indicates transcriptional starting site of GAT1 in neonatal mouse cerebral cortex and adult mouse brain. Vertical arrow 2 indicates transcriptional starting site of GAT1 in neonatal mouse intestine. Sixteen nucleotides following the transcriptional starting sites are listed. P1 and P2 are the primers used in 5' RACE for GAT1.

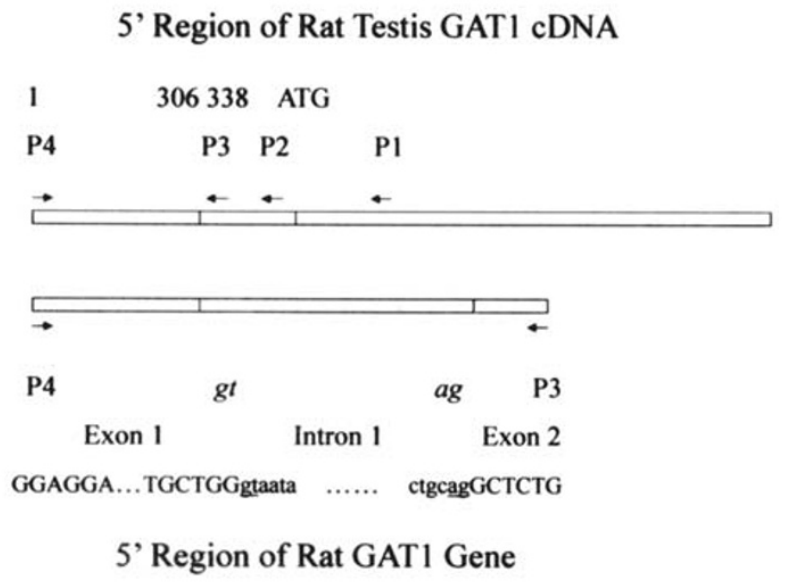

Fig 3. Diagram of 5' region of rat testis GAT1 cDNA and rat GAT1 gene Primers, P1- 4, are shown. The size of intron 1 is 783bp. End nucleotides of exon1, intron1 and exon2 are listed.
GAT1 in Day1 mouse kidney, and the product had no relation with GAT1, parallel to other' s work on adult mice[6],[7]. Tissue specific difference of GAT1 transcriptional starting sites represents the accurate tissue specific regulation of GAT1 gene expression, so that GAT1 can fulfill its specific functions in these tissues, and the abnormality of GAT1 expression has a certain connection with some diseases. Our work will help to understand the mechanism of the regulation of GAT1 gene expression in normal and pathological tissues.

\section{ACKNOWLEDGEMENTS}

We thank Ms. ZHOU Wei Ying for her technique help in cloning, Mr. JIANG Xiao Dong and Ms. HUANG Qin for their technique help in automatically sequencing on ABI 310 and Dr. HU Jing Hua for his kind help in the experiments.

This research was supported by foundations from Chinese Academy of Sciences and Special Funds for Major State Basic research of China (G1999053903).

\section{REFERENCES}

[1] Guastella J, Nelson N, Nelson H, et al. Cloning and expression of a rat brain GABA transporter. Science 1990; 249:1303-6.

[2] Ma YH, Hu JH, Zhou XG, et al. Gamma-aminobutyric acid transporter (GAT1) overexpression in mouse affects the testicular morphology. Cell Res 2000; 10:59-69.

[3] $\mathrm{Hu} \mathrm{JH}, \mathrm{He} \mathrm{XB}$, Yan YC. Identification of gammaaminobutyric acid transporter (GAT1) on the rat sperm. Cell Res 2000; 10:51-8.

[4] Sambrook J, Fritsch EF, Maniatis T. Molecular cloning: A laboratory manual. Cold Spring Harbor Laboratory Press 1989.

[5] Liu QR, Mandiyan S, Nelson H, et al. A family of genes encoding neurotransmitter transporters. Proc Natl Acad Sci USA 1992; 89:6639-43.

[6] Fei J, Huang F, Ma YH, et al. Characterization of 5'proximal sequence of mouse GABA transporter gene (GAT-1). Cell Res 1997; 7:61-7.

[7] Ma YH, Zhou XG, Duan SH, et al. Overexpression of gaminobutyric acid transporter subtype I leads to cognitive deterioration in transgenic mice. Acta Pharmacol Sin 2001; 22:340-8. 